\title{
Membrane Vesicle - A Universal Mechanism Functional Transport in the Digestive System
}

\section{Morozov IA ${ }^{1 *}$ and Tkachenko PE ${ }^{2}$}

${ }^{1}$ Chumakov Federal Scientific Center for Research and Development of Immuneand-biological Products of Russian Academy of Sciences, Russia

${ }^{2}$ Sechenov First Moscow State Medical University, Moscow, Russia

*Corresponding Author: Morozov IA, Chumakov Federal Scientific Center for Research and Development of Immune-and-biological Products of Russian

Academy of Sciences, Russia. E-mail: moroz38@gmail.com
Received: August 11, 2020

Published: September 17, 2020

(C) All rights are reserved by Morozov IA and Tkachenko PE.

\section{Abstract}

The article describes the mechanism and principle of the use of membrane vesicles in the implementation of the main functions of these organs: secretion of hydrochloric acid by parietal cells, absorption of nutrients in the small intestine and liquid at concentration bile by epithelial cells of the gallbladder. Evidence is given of the intracellular formation of hydrochloric acid in the tubulovesicles of parietal cells and the turnover of its secretory membranes during the secretory cycle, which ensures their multiple use and explaining the extraordinary lifespan of these unique cells. On this basis, a reliable mechanism of inhibition of $\mathrm{HCl}$ secretion by $\mathrm{H}+-\mathrm{K}+-\mathrm{ATPase}$ blockers are described. The article discusses in detail the endocytic mechanism of absorption of ions and nutrients by enterocytes. The mechanism of participation of the apical contractile complex of brush borders of epithelial cells in the initiation of endocytosis and cytoplasmic microtubules in the transport of membrane vesicles through the cytoplasm is analysed in detail.

Based on our own research and numerous works in the world scientific literature, it was concluded that there are two energydependent modes of transport in the absorptive epithelium of the digestive organs - transmembrane (ionic and nutritional) homeostasis, carried out by the ATPase system of the basal plasmalemma, and vesicular (endocytic), implemented by the apical complex edges and microtubules of the cytoplasm. Both modes of transport are interconnected and are under constant cellular control. It is concluded that this is directly related to the majority of cells, including those that secrete various substances: hydrochloric acid by parietal cells, enzymes by the main cells of the stomach glands and exocrinocytes of the pancreas, hormones by endocrine cells of the APUD system and, finally, mediators by nerve cells...

To clarify the problem of cell transport, it is proposed to distinguish between two types of them: homeostatic and functional. The first is carried out mainly in relation to ions on the basolateral membrane with the help of its ATPase systems and provides intracellular homeostasis and nutritional co - transport, while the second (functional) determines the performance of the main specific function with the help of membrane vesicles.

Keywords: Cell Transport; Endocytosis; Membrane Vesicles; Functional and Homeostatic Transport

\section{Introduction}

2013 was marked by the award of the Nobel Prize in Physiology or Medicine to three American scientists for their research on the mechanism of regulation of vesicular transport in cells. The laureates were Head of the Department of Cell Biology at Yale University
Professor James E. Rothman (biochemist), Professor of the Department of Molecular and Cell Biology at the University of California at Berkeley Randy W. Schekman (geneticist) and Professor of the Department of Molecular and Cell Physiology at Stanford University Thomas C Südhof (physiologist). Each of them because of their 
specialization made a contribution to decipher the genetic basis and molecular-biologicheskih formation mechanisms of intracellular movement and interaction with the cell membrane (membrane) transport vesicles providing synthesized from stem cells bathrooms specific secrets (proteins, glycoproteins, hormones, $n$ neurotransmitters).

Works by RW Schekman, JE Rothman, and. TC Südhof became part of the same mosaic describing the transport system inside the cell with the participation of vesicle vesicles. They largely determined how the formation of transport vesicles is regulated and with the help of which and how they deliver molecules (substances) to the extracellular space. Without expressing doubts about the correctness of the choice of the Nobel Committee, it should be noted that the works of the laureates of the prize concern only a particular case of intracellular vesicular transport of molecules of various biologically active substances synthesized in the cell.

In the body of living beings, including humans, there are various organs, tissues and cells that perform various functions, the synchronous work of which ensures the vital activity of the body. The functioning of some cell ensembles does not fit into the Procrustean bed of the mechanisms deciphered and described in the works of the 2013 laureates.

As an example, lying on the surface, one can cite the endothelium of the capillaries, which form the blood-tissue barrier. Transport vesicles, formed on the intracapillary surface of endothelial cells, carry out the transcytosis of various ions and biological molecules into the intercellular space of the tissue to maintain the metabolic processes of specialized cells. This process, which has a nonspecific character, was named by KE Wohlfarth-Bettermann back in 1968 [1] "cytopempsis".

The differences in the mechanisms of formation of transport vesicles in the digestive organs are most indicative. This article is devoted to this issue. In it will be given basic information about the role mebrannyh vesicles in the implementation of the secretion of hydrochloric acid by the parietal cells, as well as mechanisms of endocytosis and vesicular intracellular transport processes of absorption of nutrients epithelial cells of the small intestine and liquid absorptive holetsitami gallbladder. The morphological and functional aspects of these problems were thoroughly studied by us in the period from 1972 to 2007.
Vesicular transport in parietal cells of the gastric mucosa

The main function of the gastric mucosa is the secretion of digestive enzymes, mucus glycoproteins, and several hormones. However, about half of the cellular mass of the gastric glands is represented by unique parietal (parietal) cells that produce and secrete hydrochloric acid. The very process of gastric acid secretion from the standpoint of fundamental knowledge has always aroused great interest among biologists and physicians.

When in the early 70s of the last century we began our research on the functional morphology of parietal cells, the efforts of a large number of researchers (AJ Dalton, 1951; AW Sedar, 1957; AD Hally, 1959; S. Ito, 1961; DW Fawcett, 1962 and etc.), their ultrastructural features were described in sufficient detail. However, at that time these morphological characteristics were absolutely not functionally related to the secretion process itself. Nothing was known about what intracellular changes occur in parietal cells during the secretory cycle, how chlorine (as one of the components of $\mathrm{HCl}$ ) enters the cell, what intracellular formations it is transported through the cytoplasm and how it is released from the cell. And although a sub microscopic picture of the state of the parietal cell at rest and after secretion was described, the dynamics of the transition from one state to another, that is, the dynamics of "membrane flows" and their significance, have not been studied at all. Without knowledge of these issues, the entire functional interpretation of sub microscopic changes in the secretion process cannot be considered convincing.

Parietal cells make up to $60 \%$ of the volume of the cell mass of the gastric mucosa and are the largest cells in the gastrointestinal tract. Their average diameter in humans is $25-30 \mu \mathrm{m}$. They have a pyramidal shape, a rounded nucleus located centrally and closer to the basement membrane (Figure 1). The rough endoplasmic reticulum is represented by a few small cisterns in the basal and perinuclear zone of a mature differentiated cell, which, together with a small number of mono- and polyribosomes, indicates a very low potential for protein synthesis.

An underdeveloped intracellular mesh apparatus (Golgi apparatus) is located both in the supranuclear zone and (less often) in the basal sections of the cell. The low capacity for physiological and reparative regeneration does not explain the unusually slow renewal of the parietal cell population. M. Lipkin [2] found that the lifespan 


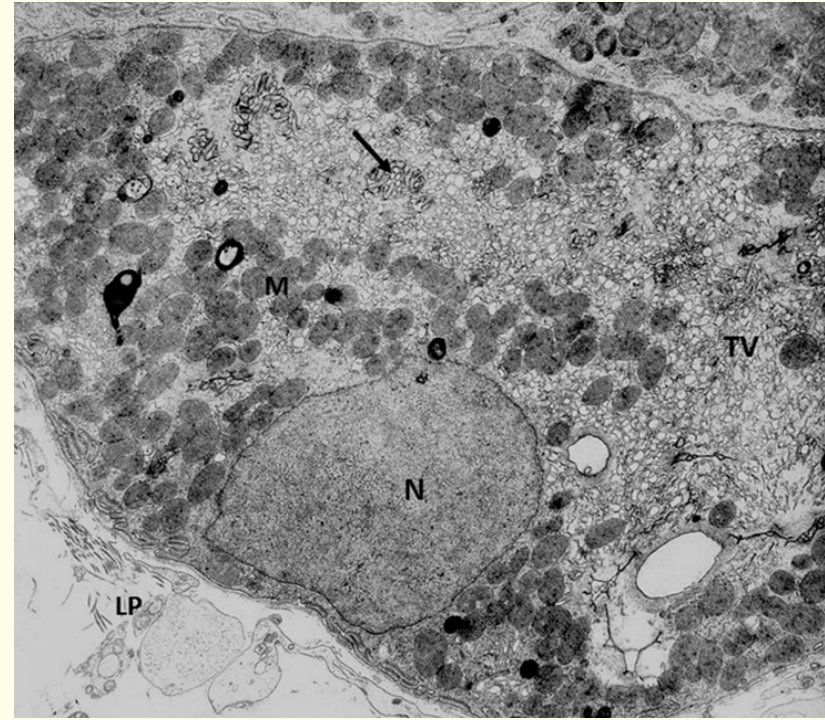

Figure 1: General plan of the structure of a mature parietal cell with a high secretory potential.

Legend: N: Nucleus; M: Mitochondria; T V: Tubulovesicles; Intracellular Tubules; LP: Lamina propria. EM, × 10000 .

of these cells in humans ranges from one year to several years. In contrast to other cells of the epithelium of the gastric parietal cells are highly developed mitochondrial apparatus. Large mitochondria up to 2 microns in length occupy up to $40 \%$ of the cytoplasm area and have a dense matrix and a huge number of cristae, which is very similar to myocardial mitochondria. In view of this parietal cells belong to the most metabolically active cells of the organism, whose mitochondrial apparatus is capable of generating and translocation of protons $(\mathrm{H}+)$ in the process of secretion of hydrochloric acid.

A distinctive feature of parietal cells, distinguishing them from all cells of the body, is the presence of a developed system of secretory membranes, consisting of intracellular tubules and a vacuolar apparatus - "tubulovesicles", which occupy about half of the cytoplasm volume during the inter digestive period. Initially, tubulovesicles were considered an element of a smooth endoplasmic reticulum. However, later, in 1960-1965, numerous data were obtained, indicating a great similarity of their membranes to the plasmalemma. No functional explanation has been found for this fact.

The plasmalemma surrounding the parietal cells has microvilli on the apical surface like those on the secretory tubules. On the lateral surfaces of the cell, the plasma membrane has interdigitation - finger-like mutual pressure of two adjacent cells. On the basal areas of the plasmalemma, there are double-circuit folds, the development of which can vary widely depending on the state of the cell [3]. According to DC Pease [4], these folds are a characteristic feature of cells related to water transport.

The possibility of comparing the ultrastructure of non- secreting gastric lining cells and at the height of secretion has been used by dozens of researchers. But HF Helander (1962-1974) and A. W. Sedar (1957-1965) dealt with this issue most seriously. It was found that in the interdigestive period, the accumulations of tubulovesicles are located, as a rule, near the apical surface of the cell and near the membranes of the intracellular tubules. When gastric secretion is stimulated, the membranes of the tubulovesicles merge with the membrane of the intracellular secretory tubules, forming numerous microvilli of various sizes.

In 1962, AW Sedar [5] published a large summary article on the ultrastructure of stimulated parietal cells. He established the complete identity of the glycoproteins of the microvilli glycocalyx with the acidic "mucopolysaccharides" of the inner surface of the tubulovesicle membrane. On this basis, he cautiously expressed the idea that the cytoplasmic vesicles (tubulovesicles) play some role in the secretory processes of the parietal cells.

AW Sedar suggested that tubulovesicles are formed by budding from finger-like depressions between the microvilli of the plasma membrane or secretory tubules and, due to reverse pinocytosis, appear in the cytoplasm, and the contents of the tubulovesicles are formed as a result of transmembrane transport of ions from the cytoplasm using carriers. Since the conclusions of AW Sedar did not fit into the carbohydrase theory of hydrochloric acid secretion, which was fashionable at that time, it is safe to say that he anticipated the emergence of the theory of the proton pump and the existence of the $\mathrm{H}+/ \mathrm{K}+-$ ATPase. The weakness of the conclusions of AW Sedar was the assertion of the existence of "reverse pinocytosis", since in this case the contents of the tubulovesicles will be captured from the lumen of the gland, which is biologically impractical.

First of all, we were interested in the problem of chlorine transport (as one of the components of hydrochloric acid) and the possible participation of the system of secretory membranes of the parietal cell in this transport. Among the researchers involved in the secretion of hydrochloric acid, the prevailing opinion was about the usual transmembrane diffusion of chlorine. This conclusion 
was made based on an almost equal concentration of chlorine in the blood plasma (120-150 meq/l) and in the lumen of the stomach (150-170 meq/l). However, the fact that in the cytoplasm of the parietal cells themselves the chlorine concentration is 10-15 times lower and does not exceed 10-15 meq/L was overlooked [6]. Therefore, it could be assumed that the pathways of chlorine movement are isolated from the hyaloplasm and its concentration cannot be measured by existing methods. Such a closed place for localization of chlorine can be precisely tubulovesicles. There was even a morphological basis for this assumption in the form of a single work by N. Pipan [7], in which the presence of a reaction product (silver chloride) in the tubulovesicles of parietal cells of a newborn mouse was shown using the histochemical method of H. Komnick and electron microscopy. And although the reaction specificity, and its stability has been criticized, completely ignore such results was not possible. In addition, no one could repeat this work. Paying special attention to the most careful observance of the reaction conditions - the freshness of the substrate (silver lactate), temperature $\left(0-1^{\circ} \mathrm{C}\right)$, inactive (red) lighting in the room and constant stirring for 2 hours, we managed to obtain amazing quality results (Figure 2) [8].

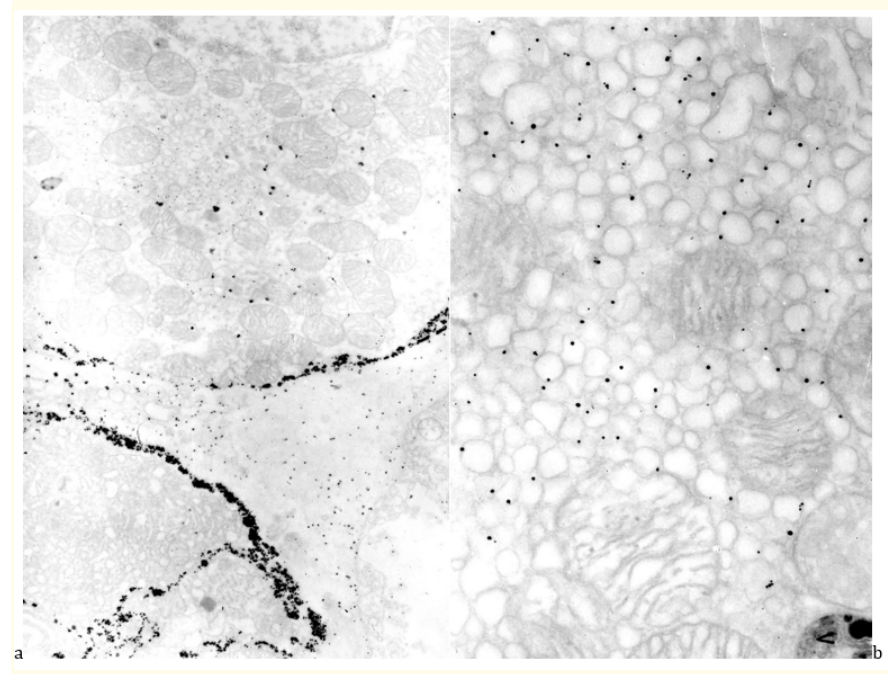

Figure 2: Histochemical study of chlorine in parietal cells of C57 Black mouse: the concentration of the reaction product on the basal plasmalemma of glandulocytes. EM, $\times 5000$; b) The reaction product in tubulovesicles. EM, $\times 20000$. The drug is not contrasted.
The reaction product in the form of silver chloride was evenly distributed in the lamina propria, in the vessel wall and on blood plasma proteins. On the basal plasmalemma of all epithelial cells and in the intercellular spaces (Figure 2 a), a pronounced concentration of the product of the histochemical reaction is determined. In the parietal cell at higher magnification, silver chloride crystals are visible only in tubulovesicles, more often on the inner surface of the membrane (Figure $2 \mathrm{~b}$ ). Freely in the cytoplasm of parietal cells and other epithelial cells of the gastric mucosa, the reaction product is rarely found, which corresponds to a low intracellular concentration of chlorine. However, the very detection of chlorine in tubulovesicles does not indicate the path by which it enters the cell and is transported in it.

To solve this issue, we decided to use the introduction of the chlorine isotope $\left(\mathrm{Cl}^{-36}\right.$ with a specific radioactivity of $\left.140 \mu \mathrm{K} / \mathrm{g}\right)$ in the form of sodium salt intraperitoneally or with food to Wistar rats, followed by the identification of the isotope using a direct method of visualization of radioactive isotopes with solutions of gold and silver halides [9]. The number of silver granules with a size of 20-100 $\AA$, formed above the site of the isotope, with this method of chlorine verification is significantly lower than with histochemistry. This is determined by limiting the amount of chlorine isotope injected by the concentration level of saline and then mixing with the total internal pool of chlorine in the body.

However, the number of "marks" was sufficient to reveal the details of the processes occurring with the chlorine ion (Figure 3). Cl- ${ }^{36}$ isotope marks were located on the outer (extracellular) side of the basal plasmalemma in the layer of glycoproteins covering this membrane. As a rule, crystals are very close to the cell membrane. In addition, they are determined between the membranes in the zone of double-contour folds of the basal plasmalemma and in the vesicles-tubulovesicles budding from these folds (Figure 3 a).

And if the moment of separation of the bubbles due to the high speed of the process is impossible to catch, then in the bubbles themselves, located not far from the folds, the mark was found quite often (Figure $3 b$ ) on the inner surface of the membrane.

On the basis of these results and the obtained picture of the distribution of the products of the histochemical reaction and isotope labels, we assumed that chlorine ions, leaving the vascular bed of the capillaries of the lamina propria of the gastric mucosa, bind 


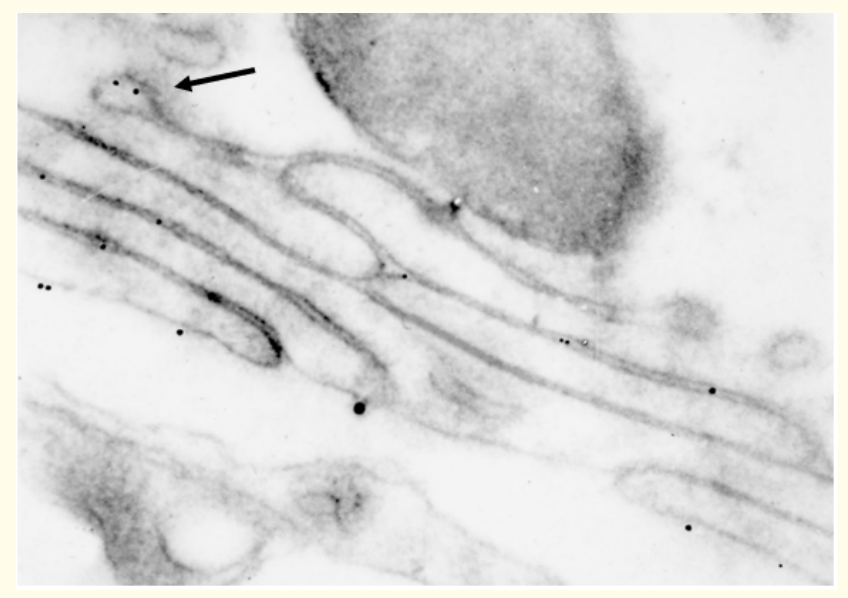

Figure 3: $\mathrm{Cl}-36$ isotope in the parietal to the tap hole. Isotope on a basal membrane in its combi folds and in vesicle budding (arrow).

The drug is not contrasted

to an acceptor located in the glycoprotein layer of the basal plasmalemma of parietal cells. Then, by the "membrane flow", which apparently occurs during the secretory cycle, chlorine ions are involved in numerous invaginations (double-circuit folds) of the cell membrane. From these folds, the constantly observed budding of vesicles occurs, in which the chlorine ion appears together with the acceptor on the inner side, isolated by the membrane of the tubulovesicle from the hyaloplasm of the cell. Tubulovesicles, accumulating in the cell, gradually concentrate in the apical part of the cell and around the intracellular secretory tubules [8].

To finally prove the possibility of interconversion of membranes of parietal cells it was possible by their morphometric analysis during the entire secretory cycle. Taking into account the error of most researchers who studied the phases of the secretory cycle in rodents (with a continuous type of secretion), we undertook an experimental test of the questions posed on a dog with an isolated ventricle according to Pavlov and obtaining a biopsy material through a fistula. The experiment on stimulation of secretion by intravenous administration of pentagastrin at a dose of 10 $\mathrm{mg} / \mathrm{kg}$ body weight was performed in this dog three times with an interval of two weeks. Biopsies of the mucous membrane of the isolated ventricle were taken in a fasted state and 1 and 3 hours after stimulation [10].

Electron microscopic examination of biopsies in the presecretory period revealed that parietal cells contain in the cytoplasm

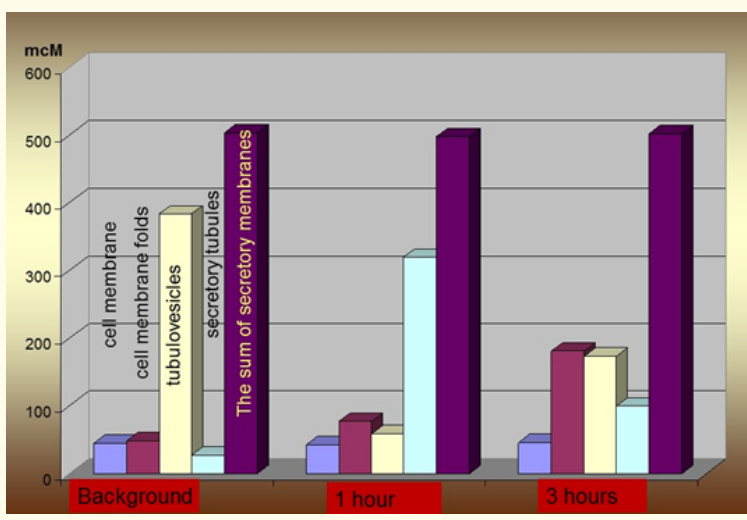

Figure 4: Results of morphometric analysis of the length of the plasmalemma and secretory membranes of parietal cells in different phases of the secretory cycle.

many tubulovesicles located around the secretory tubules and in the apical part of the cell. The membranes of these formations had a small length with rather rare microvilli (Figure 4). The lateral plasmalemma was smooth, while the basal plasmalemma had rare double-contour folds of small length.

At the height of secretion 1 hour after stimulation, the number of tubulovesicles in the cytoplasm sharply decreased. As a result of the fusion of tubulovesicles with the membranes of the secretory tubules, the number of microvilli, in which the main membrane material is concentrated, significantly increased. However, almost immediately the "membrane flow" began to move membranes to the lateral and basal surfaces of the cell. This is clearly seen from the results of morphometric analysis (Figure 4) 3 hours after stimulation. During this period, the secretion of hydrochloric acid fell almost to the initial level. The number of tubulovesicles began to increase mainly in the basal sections of cells. However, they did not yet form pronounced clusters around the tubules, which looked somewhat dilated. The greatest shifts were observed in the lateral and basal plasmalemma, which formed numerous and rather extended complex double-contour folds, from which numerous vesicles were observed to bud off tubulovesicles. The formation of an excess of membranes on the lateral and basal plasmalemma in the form of double folds indicates that the movement of membranes from the microvilli of the intracellular tubules to the membranes of tubulovesicles was carried out not by "reverse pinocytosis", but by the transition to double folds of the lateral and basal cell membranes and the budding of vesicles from them, containing chlorine $[10,11]$. 
In this case, the sum of all secretory membranes changed insignificantly, which indicates a good preservation of the membrane material during the secretory cycle and the possibility of multiple use of secretory membranes in subsequent cycles of the secretory activity of parietal cells.

This phenomenon is extremely important for such a long-lived cell, which has an extremely low potential for protein synthesis to restore the integrity of cellular organelles.

Previously, it was believed [12] that $\mathrm{H}+/ \mathrm{K}+$-ATPase, which provides proton transport, is active only if the intracellular secretory tubules are in the membrane. The inhibitor of the hydrogen pump, in order to integrate into the $\mathrm{H}+/ \mathrm{K}+$-ATPase on the membrane of the intracellular tubules and exert its blocking effect, must twice overcome the membrane impermeable to such compounds - the basal and apical.

If it is in the membrane of tubulovesicles, then it is inactive, and the inhibitor does not act on it.

At the same time, $\mathrm{H}+/ \mathrm{K}+-$ ATPase is very clearly detected in all these secretory compartments using immunocytochemistry (Figure 5). Based on the results of our study of the formation of hydrochloric acid in the parietal cell, we can make a well-grounded assumption that the generation of the missing component of the acid - a proton $(\mathrm{H}+)$ occurs during metabolic processes in mitochondria, and its translocation into the tubulovesicles is carried out with the help of $\mathrm{H}+/ \mathrm{K}+$-ATPase, built into all secretory membranes of the parietal cell, during the movement of vesicles to the secretory tubules at the time of their passage through the mitochondrial "sieve" (Figure 6).

We would never dare to assert the above, if we did not know that there are indirect data indicating that tubulovesicles in the supranuclear zone of the parietal cell contain ready-made hydrochloric acid. The Polish scientist-histologist M. Ostrouch back in 1936 [13] established that when a $1 \%$ solution of neutral red is administered intravenously to a rabbit, it accumulates in parietal cells. Moreover, in the basal part of the cells "vacuoles" (tubulovesicles) have a pale orange color, and as they move through the cytoplasm into the supranuclear zone they acquire a red coloration, that is, their contents become acidic. The experiment was successful since the neutral red molecule consists of a colouring cation and a chlorine anion, which bind to the chlorine acceptors of the basal plasmalemma. The dye travels a path like chlorine ions and

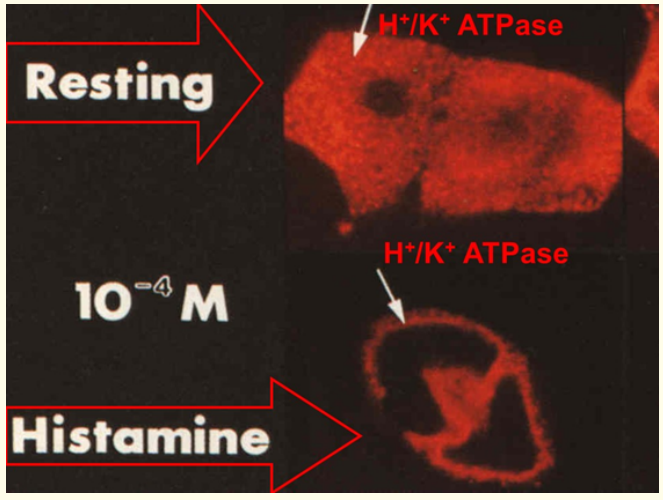

Figure 5: Distribution of products of the immunochemical reaction to $\mathrm{H}+/ \mathrm{K}+-\mathrm{ATPase}$ at rest (in all compartments) and after secretion stumulation (in cell membranes and secretory tubules).

By IM Modlin [12].

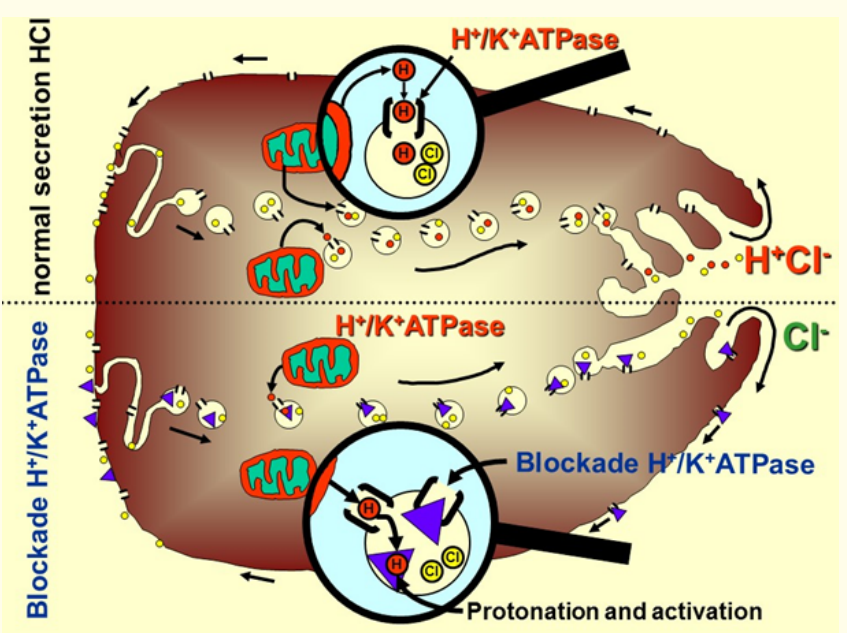

Figure 6: Diagram of the mechanism of secretion of hydrochloric acid by the parietal cell and action blockers of $\mathrm{H}+/ \mathrm{K}+-$ ATPase.

Explanations in the text.

has an affinity for cells that actively transport chlorine, that is, for the parietal cells of the stomach. The cited work testifies that the proton (as an element of hydrochloric acid) is translocated into the tubulovesicle at the time of their passage through the mitochondrial "sieve" on the way to the intracellular secretory tubules, and the tubulovesicles accumulated around the secretory tubules in the presecretory period already contain in their lumen ready for secretion hydrochloric acid... 
Finds its explanation and From this standpoint the mechanism of action of proton pump blockers (Figure 6), in which structure includes chlorine anion, thereby blocker binds to the acceptor basal plasmalemma and is in its folds and double-circuit track inside tubulovesicular. When the first hydrogen ions enter the vesicles, the protonation and activation of the $\mathrm{H}+/ \mathrm{K}+$-ATPase blocker occurs, which stops the flow of the proton into the tubulovesicles. It is important that the blocker of $\mathrm{H}+/ \mathrm{K}+-$ ATPase blocks the accumulation of hydrogen ions inside the tubulovesicles and prevents the formation of acid, without affecting the process of secretion (including chlorine) and membrane recirculation.

Thus, when considering the problem of vesicular transport in parietal cells during their secretion of hydrochloric acid, we are dealing with the transcellular transfer of vesicles (cytopempsis) and their specific contents when performing the main function. That functionality combines the processes of vesicular transport in the parietal cells in the capillary endothelium, epithelium of the proximal tubule with those that occur in a large number of cells, sinteziru- guides and secreting substances and compounds mostly of protein nature, which deals with the Nobel laureates in Physiology or Medicine 2013 the year... Despite the fact, that in both cases we are dealing with a vesicular transport regulation mechanisms of these two groups are fundamentally different, if only so that we describe the case of parietal cells is absolutely no participation of the rough endoplasmic reticulum and the Golgi apparatus in the formation of secretion and transport vesicles... The microvilli of the intracellular secretory tubules do not have the classic structure of the brush border, but are, in fact, an excess of membranes formed as a result of the fusion of the tubulovesicle membranes with the tubules, which is subsequently transported by mobile clusters of the lateral plasmalemma to the double-circuit folds of the basement membrane. A new population of tubulovesicles buds from them to implement the next secretory cycle of the parietal cell.

Vesicular transport in absorptive enterocytes of the small intestine and gallbladder

The main function of the epithelial cells of the mucous membranes of the small intestine and the gall bladder is the absorption (absorption) that is fundamentally ex and chaet these cells from the above parietal cells, is secreted. In the early 1960s, through the efforts of a group of scientists led by A. M. Ugolev, parietal (membrane) digestion was discovered [14]; the concept (hypothesis) of transmembrane transfer of substances through the brush border of enterocytes into the cytoplasm of the cell [15] using an enzymetransport complex consisting of a hydrolytic enzyme, a transporter (channel), and an energizer (ATPase) [16] was established. At the same time, a huge number of hypotheses about the mechanisms of transmembrane transfer appeared. It should be noted that all of them were based on the results of experimental work carried out in vitro with the use of isolated cells and inverted and nevyvernutyh segments of the small intestine small laboratory animals. The hypotheses were based on the prevailing ideas about the transport of substances through the plasma membrane of non-polar somatic cells, completely immersed in the internal environment of the body. These mechanisms, associated with the functioning of transport ATPases and direct transformation of the energy of macro ergs into the work of transferring substances from the intercellular space to the cell and vice versa, were extrapolated to the polar epithelium of the small intestine. The apical surface of enterocytes, although covered with a supraepithelial layer of mucous overlays, is still open into the lumen of the small intestine, communicating with the external environment. After establishing the absence of transport $\mathrm{Na}+-\mathrm{K}+$-ATPase in the apical plasmalemma of epithelial cells of the small intestine, a scheme was proposed for the secondary energization of absorption mechanisms due to processes occurring on the basolateral membrane of epithelial cells [17], in which various ATPases are present. Naturally, the transmembrane transport of substances from the intestinal lumen into the cell should cause an increase in the concentration of the transported substance in the cytoplasm. However, this phenomenon is absent in experiments in conditions in situ, and in vivo: the concentration of any of the substances being transported into the cytoplasm of enterocytes not increased.

This situation led AM Ugolev in one of his early works to an unambiguous conclusion [18]: "Perhaps in the intestinal cells there is such a specialization that substances penetrating from the base side enter the cytoplasm of cells, and substances coming from the intestinal cavity move extra plasmically", meaning "channels of ergastoplasm (intracellular channels) and intercellular spaces". The author left out of sight the endocytic (pinocytosis) mechanism, the chain of events in which (transcytosis) can be considered as a channel of extraplasmic transport. Despite the existence of objective evidence of the existence of endocytosis in the epithelial cells of the small intestine, it was during this period, thanks to the experimentally unfounded conclusions of SL Clark [19,20], among researchers the opinion that nutrients are absorbed by pinocytosis in mammals only during milk feeding (that no one was not denied), and pino- 
cytosis, both in speed and in volume, cannot meet the metabolic needs of an adult. Thus, a paradoxical situation has developed: the objectively observed pinocytosis in epithelial cells of the small intestine is rejected as a possible absorption mechanism.

Starting our research, we first of all paid attention to the complete disregard for the presence of the supraepithelial layer of mucus in the schemes of the digestive-transport processes in the small intestine. The combined efforts of several laboratories in Moscow, Obninsk, Pushchino, Tomsk and Tashkent have decoded not only the structure of this layer, which is based on mucins secreted by goblet cells, but also the enzyme composition, radial distribution of hydrolases, heterophasic digestion activity and resident microflora of mucous overlays [21-23]. All these results were included in the collective monograph [24], in which the concept of "parietal digestion" was introduced as one of the intermediate stages of the digestive transport conveyor between cavity and membrane hydrolysis. However, this problem is not the purpose of this publication, and we will not dwell on it in detail.

The main task of the research was to decipher the mechanisms and significance of the endocytic way of absorption of nutrients by enterocytes of the small intestine. We have undertaken a number of in vivo and in situ experiments with the introduction of various substances into the stomach of animals through a tube, or directly into the intestine. We used ${ }^{45} \mathrm{CaCl}_{2}[25],{ }^{58} \mathrm{Co}$-cyanocobalamin, ${ }^{14}$ C-thiamine, ${ }^{3} \mathrm{H}$-tocopherol, ${ }^{3} \mathrm{H}$-retinol, ${ }^{3} \mathrm{H}$-cholesterol, horseradish peroxidase [26], ferritin, as well as $\beta$-lactoglobulin and bovine serum albumin conjugated with horseradish peroxidase.

As a result of the study and analysis of the experimental material obtained by morphological methods using histochemistry and the direct method of identification of radioactive isotopes, it was found that the fundamental difference in the localization of ions $\left({ }^{45} \mathrm{Ca}^{-2}\right)$ (Figure 7a) and large molecular substances (peroxidase, etc.) (Figure $7 \mathrm{~b}$ ) in the cell structures in the process of absorption and transport through the enterocyte is not. Thus, during the first minutes after the introduction of substrates into the small intestine, the test substance was sorbed in the structures of the parietal mucus layer and on the apical surface of enterocytes. At the beginning of absorption, the absorbed substances were found in endocytic vesicles, and already 3-5 minutes after their introduction into the intestine, their appearance in the intercellular space below the zone of close contact was noted. The release of substances from the transport vesicles into the intercellular space was carried out

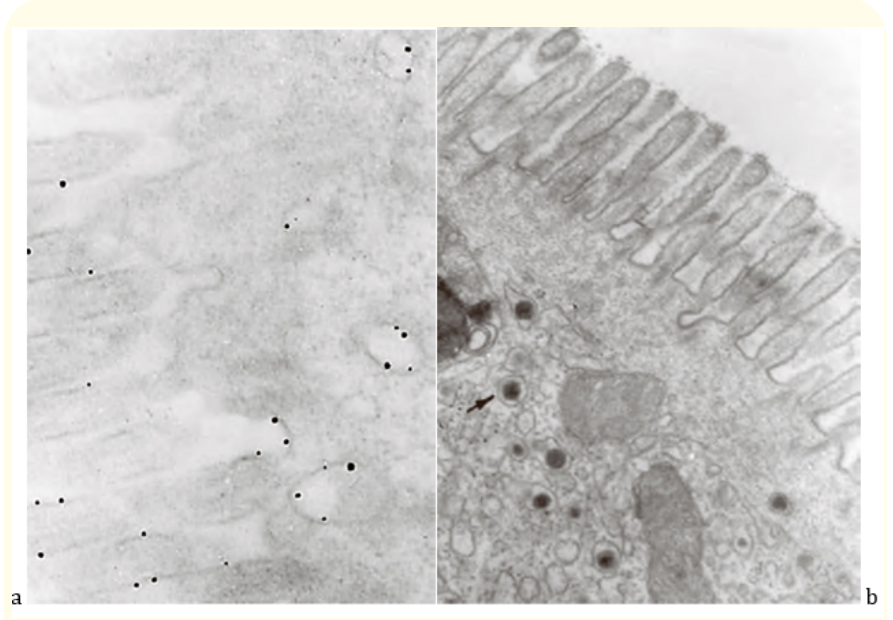

Figure 7: a). Endocytotic absorption isotope and ${ }^{45} \mathrm{CaCl}^{2+}$ after administration in the Ludok is disposed on the surface of microvilli in endocytotic vesicles. The drug is not contrasted. EM x 40,000. b)

Peroksidaze in endocytotic vesicles (arrow). EM, $\times 20,000$.

by exocytosis. It should be noted that we did not find transmembrane transfer of substances directly into the cytoplasm of cells, with the exception of single cells of the apical pole of the villi with signs of involution, completing their life cycle and subject to extrusion from the epithelial layer.

We observed a similar picture in the case of natural short-term (15 minutes) feeding of rats with a mixed food consisting of white bread, milk, and sunflower seeds in a homogenized form $[27,28]$. Already 25 minutes after feeding, a significant amount of nutrients were observed in the suction cells of the duodenum and jejunum, mainly in the upper third of the villi, which were in the vesicular structures (Figure 8a). merged with the lateral portion of the observed fusion of transport vesicles, the formation of larger vacuoles, which together with the vesicle membrane s enterocytes dumped their contents into the extracellular space. Here there was a temporary deposition (Figure 8b) of absorbed nutrients (food particles and chylomicrons), and then they passed through the basement membrane and were found in the lamina propria and around the capillaries.

When studying the control group of sexually mature rats before the experiment with natural feeding, starving for 24 hours under conditions excluding coprophagia, signs of vigorous activity of the small intestine were obtained. They were expressed in the pres- 


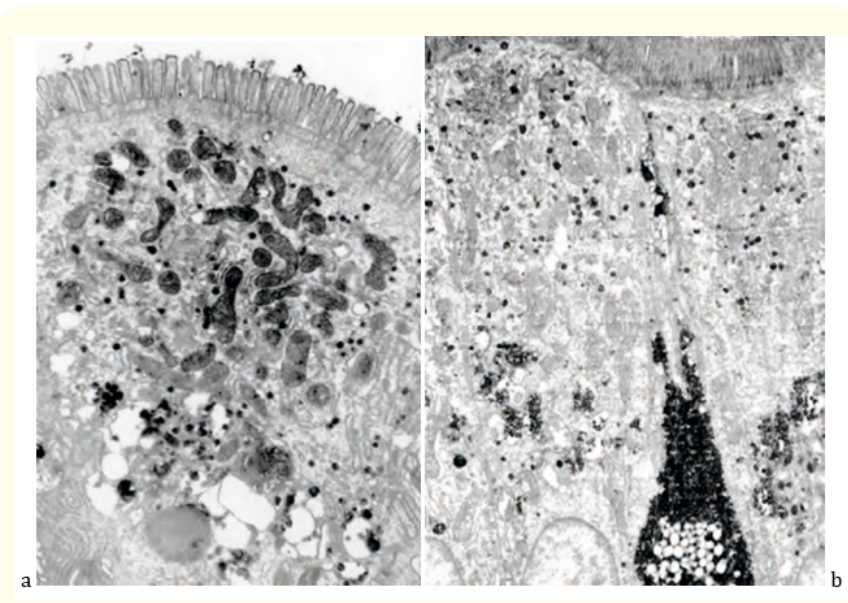

Figure 8: Absorption of nutrients after natural feeding: a) food particles in endocytic vesicles. EM, × 10,000; b) Large vacuoles with nutrients in the supranuclear zone and the accumulation of food particles in the intercellular space. $\mathrm{EM}, \times 8000$.

ence of a large number of endocytic vesicles in the apical part of the enterocyte, some of which contained osmiophilic electron-dense particles, which were found to be bile micelles, the main transport form of bile [29]. As this is not surprising, since the rat is a rodent with a permanent type of secretion, in which for the same missing gallbladder. All aspects of the digestive-transport processes in the small intestine are described in detail in our monograph [30].

The mucous membrane of the gallbladder is covered with villi, the peculiarity of which lies in their strong tortuosity with the presence of numerous bays and invaginations of various sizes and depths. The height of the villi is variable, but comparable to the villi of the duodenum. Their shape is leafy and, when viewed from the surface, is very similar to the shape of the villi of the duodenal mucosa. The villi are covered with a single-layer columnar epithelium with a well-defined brush border. Goblet cells in the epithelium (despite their mention by some authors) are absent. This determines the absence of the formation of the parietal layer of mucus in the gallbladder, the complete openness of the superficial epithelium and its availability of bile. In this lies one of the main differences between the mucous membranes of the gallbladder and small intestine.

The ultrastructure of the epithelial cells of the gallbladder almost does not differ from nterocytes. At the same time, the epithelial cells of the bladder mucosa are somewhat smaller than the enterocytes, and the microvilli are also shorter. The main function of epithelial cells of the mucous membrane of the gallbladder is to concentrate bile because of absorption (absorption) of water and molecular components dissolved in it. Due to this, the same endocytosis processes are observed in cholecytes as in enterocytes, and the formation of transport vesicles with electron-transparent contents (Figure 9a). At the same time, in a normal gallbladder, bile micelles are not captured by endocytic vesicles.

As noted above, on the apical surface of the epithelial cell, endocytic invaginations and vesicles are formed, which involve water-soluble elements in the aqueous phase of bile - various ions, free cholesterol, and its esters. It is possible that in this case those elements for which there are binding acceptors, anionic and cationic sites of glycoproteins and apolipoproteins in the structure of the glycocalyx are more actively absorbed. The formed transport vesicles move to the lateral surface of the cell and, merging with the membrane, pour out their contents into the intercellular space. This process is active, consumes energy and can carry out antigradient transfer of various substances. Lateral membranes in the supranuclear zone of the epithelial cell have numerous folds, which are considered by most researchers as interdigitations that hold together adjacent cells (Figure 9a). In fact, these folds are a kind of saccular protrusions formed by an excess of membrane material brought here by endocytic vesicles.

In the gallbladder, under normal conditions, the suction epithelium provides only one concentration function, and the volume of the absorbed fluid is not as large as in the small intestine. Due to this evolutionary development, there has been a lack of specialization of absorbent epithelial cells of the gallbladder, which have direct contact only with bile. At the same time, with cholesterosis of the gallbladder, the pathogenesis of which lies in general disorders of lipid metabolism and the consumption of large amounts of animal food, the epithelium faced an inadequate supramolecular complex in the form of bile micelles, which it cannot quickly move in the vesicle to the lateral membrane. As a result, in the supranuclear zone, transport vesicles merge, forming large vacuoles with a huge number of bile micelles (Figure 9b). Micelles and lipid droplets inside the vacuole do not have a delimiting membrane, and the vacuole itself has a very thin elementary membrane that separates its contents from the cell cytoplasm. Subsequently, these vacuoles merge with the lateral membrane and pour out their contents into the intercellular space. Details of the processes occurring in cholesterosis of the gallbladder are described in our monograph [31]. 


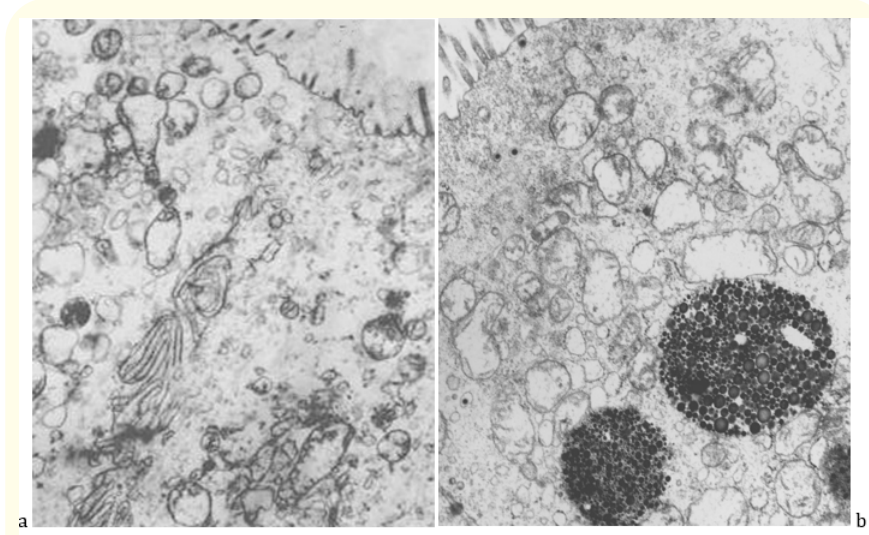

Figure 9: The ultrastructure of the mucous membrane of the gallbladder: a) endocytic vesicles in the cholecitis of the bladder without signs of cholesterosis; b) bile micelles in endocytic vesicles and as part of a conglomerate of merged vesicles in cholesterosis. Explanations in the text. EM, a - x 5000, b - x 8000.

Comparison of the morphological patterns of endocytosis in the epithelium of the small intestine and gallbladder revealed a significant difference in the structure of absorbed substances. The small intestine actively absorbs through endocytosis not only water and ions, but also all classes of macro- and micronutrients in monomeric form. The lipids are absorbed not only in the form of monomeric components (Ms p GOVERNMENTAL acids, glycerol, and $t$. D.) But also in the form of droplets of triglycerides, but also chylomicrons which are formed in the cavity of the small intestine of the biliary micelles, soluble in itself hydrolysis products of dietary fat... Pictures of endocytic transport of bile micelles are especially clearly detected in the duodenum during the interdigestive period, which was described by us $[29,30]$ in the study of enterohepatic recirculation of bile.

When studying the first reaction of the brush border of enterocytes after natural feeding of experimental animals, it was found that there is no increase in the length of microvilli and an increase in the area of the apical membrane to enhance membrane hydrolysis and transmembrane transport [15]. On the contrary, already in the early stages, microvilli are significantly shortened, and their membrane acquires a wavy shape. It was natural to assume that such a reaction was caused by the activation of the apical contractile complex of microvilli, the structure of which has been studied in detail by several researchers [32,33] (Figure 10). A. M. Ugolev

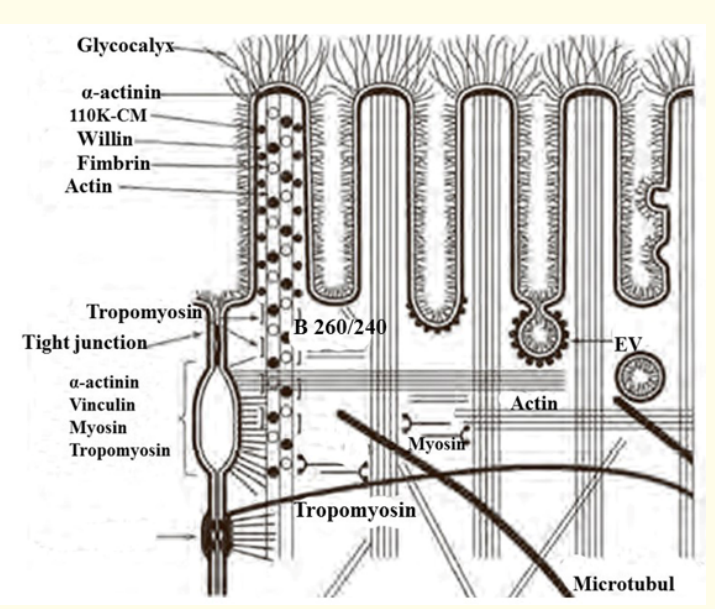

Figure 10: Diagram of the organization of the apical contractile complex of absorptive epithelial cells of the small intestine.

believed that this complex causes active dropping of the glycocalyx in order to renew it: "Rapid renewal of the glycocalyx ensures the effective functioning of the brush border as a porous reactor, since by dropping the" mature "glycocalyx, a peculiar effect of constant cleansing of pores or intervillous spaces is created" [17]. Such a statement would be valid if the life of the enterocyte was not so short (3-4 days), and the meeting of the intestinal cell with nutrients was not limited to its location in the upper third of the villus for only one day.

We decided to test the possibility of the involvement of the apical contractile complex and the cytoskeleton of enterocytes in the absorption and intracellular transport of endocytic vesicles using inhibitory analysis. For this purpose, we used cytochalasins B and D, which inhibit actin polymerization, and colchicine, which binds to tubulin and stops the assembly of cytoplasmic microtubules from the $(+)$ - end. Inhibitors were administered intraperitoneally to hungry rats 20-30 minutes before feeding. The control group was injected with saline. The methodological details of the study can be found in our publication [34]. After short-term feeding, the small intestine of the animals of all three groups was studied after 30 and 60 minutes, as well as after 3-4 hours. Already in the early stages after feeding, when studying semi-thin sections, the effects of inhibitors were revealed: in the control group, food substances were observed in the supranuclear zone and in the intercellular spaces, in the group with cytochalasin, food absorption was absent 
(Figure 11a), and in the group with colchicine food substances accumulated in the supranuclear zone, but did not penetrate into the intercellular space. Electron microscopy revealed these processes more clearly, especially in the experimental groups. Cytochalazins bind to the rapidly growing end of the actin filament, blocking the attachment of subunits at this end, and cause reversible depolymerization of microfilaments. All these properties of cytochalasins can be manifested because they are able to penetrate the cell membrane and interact with the complex of actin filaments of the enterocyte. But most importantly, they can cut actin filaments and thus completely block their function.

Cytochalasin D is considered the most active, although in our studies we did not notice any differences. The use of colchicine as an inhibitor of absorption did not lead to the cessation of endocytosis since colchicine has no effect on the actin filaments responsible for this process. On the contrary, active endocytosis and the accumulation of transport vesicles (Figure 11b) containing electron-dense particles of nutrients were observed, some of which merged into larger vacuoles. However, there was no transport of vesicles to the lateral plasmalemma and exocytosis of their contents into the intercellular space.

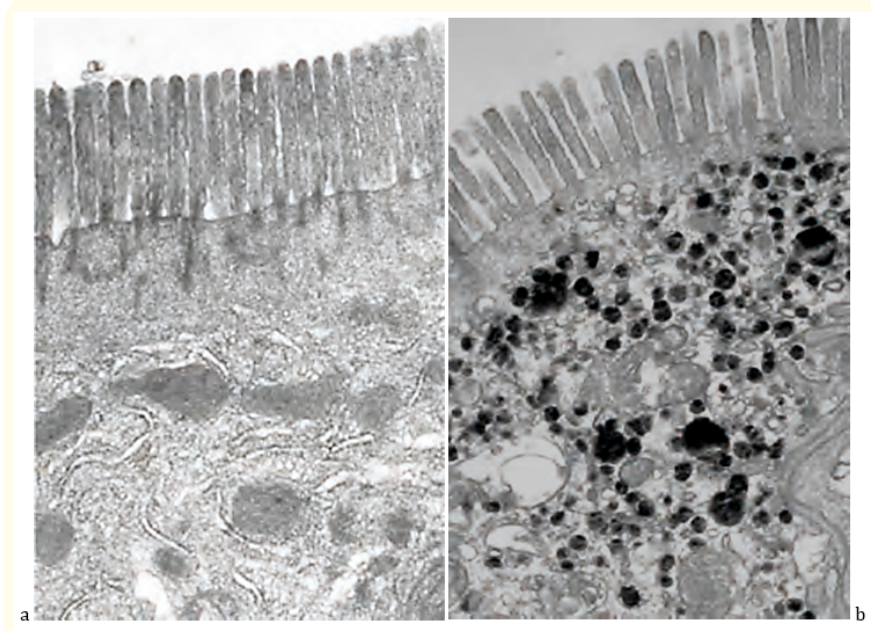

Figure 11: Changes in the ultrastructure of enterocytes upon inhibition of cytoskeleton elements: a) absence of endocytosis after inhibition by cytochalasin B; b) the accumulation of ndocytic vesicles in the supranuclear zone after colchicine inhibition. Explanations in the text. EM, $\times 15,000$.
Considering that tubulin and microtubules collected from it are the main targets of colchicine, it was concluded that microtubules are involved in the transfer of transport endocytic vesicles to the lateral plasmalemma of enterocytes. Microtubules are a permanent and indispensable component of all eukaritic cells.

In recent years it has been found that in a living cell, microtubules grow shorter, disappear, ie are constantly in dynamic instability. It turned out that the average half-life of cytoplasmic microtubules is only 5 minutes. So, in 15 minutes, about $80 \%$ of the entire population of microtubules is renewed.

In this case, individual microtubules at the growing end can elongate relatively slowly (4-7 $\mu \mathrm{m} / \mathrm{min})$ and then shorten rather quickly (14-17 $\mu \mathrm{m} / \mathrm{min}$ ). About $10-20 \%$ of microtubules remain stable for a fairly long time (up to several hours). Stabilization of microtubule associated with or modification of tubulin or with their binding to additional MAP (membrane associated proteins) and the microtubule protein with other cellular components.

The role of cytoplasmic microtubules can be reduced to two functions: skeletal and motor. The skeletal, or scaffold, role is that the location of the microtubules in the cytoplasm stabilizes the shape of the cell. However, they are required for a number of intracellular transports, such as endocytosis, exocytosis, mitochondrial movement, etc. Their motor role is not only in the fact that they create an ordered, vector system of movement.

Cytoplasmic microtubules, in association with specific associated motor proteins (MAP, cytosolic kinesins), form ATPase complexes capable of driving cellular components. Some are involved in the transfer of only mitochondria, others only transport vesicles. Many of the kinesins bind specifically to their weights and carry out their directed transfer along the microtubule axis towards its $(+)$ - end. Thus, we have proved the role of actin in the initiation and implementation of endocytic absorption of nutrients and microtubules in the movement of transport (endocytic) vesicles to the lateral membrane of enterocytes. Undoubtedly, a similar absorption mechanism is present in other absorbent cells with a brush border and implementing endocytic absorption, for example, the epithelium of the gallbladder [31] and proximal renal tubules [35]. But it is fundamentally different from the mechanisms of micropinocytosis observed in the capillary endothelium since the latter is realized without energy expenditure. 
As for the sharp scientific discussion between me and A.M. Ugolev, which was conducted for 15 years orally at the School-Seminar in Kaluga, symposia and congresses of the Society of Physiologists of the USSR, it can be noted that in scientific publications it was one-sided, since Academician A. M. Ugolev never once referred to our publications in his books and articles. Even when we proved [36] that it is impossible to study absorption processes in vitro, since even a short-term decrease in blood flow in the mesenteric artery by $50 \%$ (ischemia) leads to damage to the apical membrane and intercellular contacts, A. M. Ugolev allegorically acknowledged our correctness and wrote [17]: "Thus, the data obtained under in vitro conditions lead the researcher to the idea of systems that exist only in a damaged form. Now the difficult task arises of subjecting to a critical analysis the results obtained in the conditions of traditional acute experiments. "However, such a revision was not made in connection with the death of A. M. Ugolev.

Summing up the results of our review on the existence and mechanisms of vesicular intracellular transport in the digestive organs, it should be said that the scientific discussion about transmembrane and endocytic transport concerned only absorption processes in the small intestine and has nothing to do with general biological fundamental concepts of transport. If we concern only the transport function of the cell membrane, then there are two types of transport: transmembrane, carried out without the expenditure of membrane material, and endocytic - with the expenditure (or transfer) of cell membranes. Transmembrane transport can be active (with energy consumption) and passive (without energy consumption). Passive transport (various types of diffusion) is a rudiment from prokaryotes. It is extremely slow, low-volume, and does not play any role in the functioning of the cell. Thus, we mainly deal with two active modes of transport: transmembrane and endocytic. Back in the 1970s, evidence was obtained that in specialized epithelial cells separating different environments, the apical and basement membranes should differ from each other not only morphologically, but also functionally. Even then, most authors believed that the molecular mechanisms of ion transport are localized on the basement membrane of cells. In this direction, a huge amount of research was carried out, from which it followed that, despite the gigantic surface of microvilli of the apical membranes, the transport ATPase in them is about $5 \%$ of the sum of all transport ATPases of the cell. Moreover, the brush border contains only $\mathrm{Ca}^{2+}$-activated, $\mathrm{Mg}^{2+}$-dependent ATPase [37]. It was found that myosin of the apical contractile complex precisely possesses this activity. All this indicates that the bulk of the transport ATPases, including the integral $\mathrm{Na}+/ \mathrm{K}+$-ATPase, are apparently localized predominantly on the basal plasmalemma. They are partially used by some cellular organelles, such as the rough endoplasmic reticulum and mitochondria, carrying out not only energy-dependent transport of ions, but also co-transport from the intercellular space to the cell of monomeric sugars, amino acids and other nutrients necessary for intracellular metabolic processes.

Thus, in the absorptive epithelium of the digestive organs, we are dealing with two energy-dependent modes of transport - transmembrane (ionic and nutritive) homeostatic, carried out by the ATPase system of the basal plasmalemma, and vesicular (endocytic), realized by the apical contractile complex of the brush border. Even though vesicular transport performs the main function of the epithelium - absorption, it is interconnected with the transmembrane homeostatic transport, without which it cannot be realized.

It is important that both modes of transport are under constant cellular control. However, this postulate is directly related to the majority of cells, including those that secrete various substances: hydrochloric acid by parietal cells, enzymes by the main cells of the stomach glands and exocrinocytes of the pancreas, hormones by endocrine cells of the APUD system and, finally, mediators by nerve cells. All these cells also use vesicular transport in performing their specific function, while simultaneously carrying out homeostatic transmembrane transport, which is realized by the ATPase system.

\section{Conclusion}

Therefore, it is necessary to distinguish between two interrelated modes of transport that exist in most specialized cells - homeostasis and functional. The first is carried out mainly in relation to ions on the basolateral membrane with the help of its ATPase systems and provides intracellular homeostasis and nutritional co-transport, while the second (functional) determines the performance of the main specific function with the help of membrane vesicles.

All kinds of cell transport mechanisms (diffusion, sodium-independent transport etc.) have been discovered in vitro. We have shown for the first time in vivo that both vesicular (endocytosis and exocytosis) and transmembrane ATP-dependent transporters work functioning in polar secreting cells and absorptive cells. This fact have never been described in medical and biological papers before. 


\section{Bibliography}

1. Wohlfarth. Bottermann the K. E. Dynamik der Zelle. Mikroskopie Bd. 23.3 (1968): 71-96.

2. Lipkin M. "Proliferation and differentiation of gastrointestinal cells". Physiological Reviews 53.4 (1973): 891-915.

3. Nomura G. "On the submicroscohic morphogenesis of parietal cell in the gastric gland of the human fetus". Zeitschrift für Anatomie und Entwicklungsgeschichte 125.3 (1966): 316-356.

4. Pease DC. "Infolded basal plasma membranes found in epithelia noted for their water transport". Journal of Biophysical and Biochemical Cytology 2 (1956): 203-208.

5. Sedar AW. "The fine structure of the oxyntic cell in relaxation to functional activity of the stomach". Annals of the New York Academy of Science 1 (1962): 9-29.

6. Woodbury JW. "The cell membrane: ionic and potential gradients and active transport". Neurophysiology Ed. 2 WB Saunders Co., Philadelphia (1965): 1-25.

7. Pipan N. "Chloridnachweis in der belegzellen der maus wahrend der differenzierung". Cytobiologie 8.6 (1974): 509519.

8. Morozov IA. "Transport of chlorine ions in parietal cells of the gastric mucosa (electron-histochemical and autoradiographic study)". Bulletin of Experimental Biology and Medicine 7 (1977): 29-32.

9. Normandin D. "Direct deposition at high resolution of specific metals from solutions at radioactive sites in tissue sections". Transactions of the American Microscopical Society 92.3 (1973): 381-398.

10. Morozov IA. Morphological aspects of secretion of hydrochloric acid by the stomach in health and disease (Functional morphology of parietal cells): Author's abstract. dis.... doct. honey. sciences (1977): 31.

11. Morozov IA and Hramtsov A. "Study of morphofunctional properties of parietal cells of the stomach upon activation of secretory activity". Fiziologicheskil Zhurnal USSR them. I.M.Sechenov 65.3 (1979): 456-461.
12. Modlin I. The M. From prout to the proton pump. SchnetztorVerlag GmbH B-Konstanz (1995): 100.

13. Ostrouch M. "Histophysiologische untersuchungen uber die hauptdrusen des magens. I. Die bedentung der belegesellen im sekretionsprozess". Ztschr. Fur Zelifor-schung und Mikroskopische Anatomie 26 (1936): 424-438.

14. Ugolev AM. "On the existence of parietal (contact) digestion". Bulletin of Experimental Biology and Medicine 49.1 (1960): 1217.

15. Ugolev AM. "Organization and regulation of processes of membrane digestion and transport". Fiziologicheskil Zhurnal USSR them. I.M.Sechenov 56.4 (1970): 651-662.

16. Ugolev AM. "Membrane digestion. Polysubstrate processes, organization and regulation”. Nauka (1972): 358.

17. Membrane hydrolysis and transport: New data and hypotheses/Ed. A. M. Ugoleva. Nauka (1986): 240.

18. Ugolev AM. "Physiology and pathology of parietal (contact) digestion”. Leningrad: Nauka (1967): 230.

19. Clark SL. "The ingestion of proteins and colloidal materials by columnar absorptive cells of the small intestine in suckling rats and mice". Journal of Cell Biology 5 (1959): 41-49.

20. Clark SL. "Alkaline phosphatase of the small intestine studied with electron microscope in suckling and adult mice". Anatomical Record 139.2 (1961): 216.

21. Lysikov A and Morozov IA. "Ultrastructure of the parietal mucous layer of the small intestine". Bulletin of Experimental Biology and Medicine 11 (1990): 550-554.

22. Morozov IA., et al. "The digestive function of the supraepithelial mucous layer of the small intestine". Fiziologicheskiı Zhurnal USSR them. I.M.Sechenov 76.4 (1990): 515-522.

23. YM Galperin and P Lazarev. "Digestion and homeostasis". Nauka (1986): 304.

24. The structure and function of the mucous layer of the small intestine / Ed. I. A. Morozov. Tempus (1998): 282. 
25. Morozov IA., et al. "Study of subcellular localization of Ca 45 during its absorption by the epithelium of the small intestine of rats". Bulletin of Experimental Biology and Medicine 12 (1980): 736-738.

26. Lysikov YA., et al. "Vesicular transport of peroxidase by epithelial cells of the small intestine of adult rats". Bulletin of Experimental Biology and Medicine 4 (1983): 114-118.

27. Khvylya SI., et al. "Ultrastructural study of the reaction of microvilli of enterocytes of the small intestine of rats during natural feeding". Bulletin of Experimental Biology and Medicine 11 (1984): 624-627.

28. Morozov IA., et al. "Vesicular transport of nutrients in the small intestine with natural nutrition". Vopr. Nutrition 3 (1985): 42-49.

29. Morozov IA., et al. "Electron-microscopic study of the process of hepatic-intestinal recirculation of bile components". Fiziolohichnyi Zhurnal 71.11 (1985): 1419-1427.

30. Morozov IA., et al. "Absorption and secretion in the small intestine: submicroscopic aspects". Medicine (1988): 224.

31. Ilchenko AA., et al. "Cholesterosis of the gallbladder". GEOTARMedia (2007): 232.

32. Bretscher A., et al. "Localization of actin and microfilamentassociated proteins in the microvilli and terminal web of the intestinal brush-border by immunofluorescence microscopy". Journal of Cell Biology 79 (1978): 839-845.

33. Matsudaira PT and Burgess DR. "Identification and organization of the components in the isolated microvillus cytoskeleton". Journal of Cell Biology 83 (1979): 667-678.

34. Morozov IA and Verina TY. "Inhibitory analysis of the role of the cytoskeleton in the absorption of nutrients in the small intestine". Fiziolohichnyi Zhurnal 79.6 (1993): 48-56.

35. Apodaca G. "Endocytic traffic in polarized epithelial cells: role of the actin and microtubule cytoskeleton". Traffic 2.3 (2001): 149-159.
36. Morozov IA., et al. "Electron microscopic study of the permeability of enterocytes of the small intestine for colloidal lanthanum in experiments in vitro and in vivo". Fiziolohichnyi Zhurnal 68 (1982): 1261-1268.

37. Tashmuhamedov BA. "Active transport of ions in the plasma membranes of animal cells". Biological Membranes ed. P. V. Sergeeva. M. Medicine (1973): 248.

\section{Assets from publication with us}

- Prompt Acknowledgement after receiving the article

- Thorough Double blinded peer review

- Rapid Publication

- Issue of Publication Certificate

- High visibility of your Published work

Website: www.actascientific.com/

Submit Article: www.actascientific.com/submission.php

Email us: editor@actascientific.com

Contact us: +919182824667 